\title{
Impulsive synchronization of fractional-order complex-variable dynamical network
}

\author{
Yu Xiong ${ }^{1}$ and Zhaoyan $\mathrm{Wu}^{1 *}$ (B)
}

"Correspondence:

zhywu@jxnu.edu.cn

'School of Mathematics and

Statistics, Jiangxi Normal University,

No.99 Ziyang Avenue, Nanchang,

P.R. China

\begin{abstract}
The impulsive synchronization of a fractional-order complex-variable network is investigated. Firstly, static impulsive controllers are designed and the corresponding synchronization criteria are derived. From the criteria, the impulsive gains can be calculated. Secondly, adaptive impulsive controllers are designed. Noticeably, the impulsive gains can be adjusted to the needed values adaptively. Finally, numerical examples are provided to verify the results.
\end{abstract}

Keywords: Synchronization; Complex variable; Fractional-order network; Impulsive control

\section{Introduction}

In recent years, fractional-order differential systems have gained increasing attentions due to the fact that they can better describe the memory and hereditary properties, such as elastic systems, dielectric polarization, heat conduction, electromagnetic waves, and financial systems [1-12]. In [7-9], the authors designed some kinds of memristive hyperchaotic system and discussed their applications. In [11], the author studied a fractionalorder financial system. For those large-scale fractional-order systems, they usually contain large number of interactive individuals and are modeled by fractional-order dynamical network. The nodes denote the individuals and the edges denote the interactions among individuals. In [13], the authors considered fractional-order neural networks. In [14], the authors investigated a time-delay neural network.

Synchronization of dynamical networks has been extensively studied [15-37], with a view on power grids, unmanned aircraft operation, parallel image processing, and so on. However, due to the complexities of a dynamic network, achieving synchronization by inner adjustment is difficult and even impossible. Therefore, appropriate external controllers need to be designed. So far, many control schemes have been adopted to design suitable controllers, such as impulsive control [16, 21-32], intermittent control [38-41], pinning control [42, 43], and feedback control $[44,45]$.

In the real world, many complex systems cannot be controlled by continuous control and endure continuous disturbance. Impulsive control, as a typical discontinuous control

(c) The Author(s) 2021. This article is licensed under a Creative Commons Attribution 4.0 International License, which permits use, sharing, adaptation, distribution and reproduction in any medium or format, as long as you give appropriate credit to the original author(s) and the source, provide a link to the Creative Commons licence, and indicate if changes were made. The images or other third party material in this article are included in the article's Creative Commons licence, unless indicated otherwise in a credit line to the material. If material is not included in the article's Creative Commons licence and your intended use is not permitted by statutory regulation or exceeds the permitted use, you will need to obtain permission directly from the copyright holder. To view a copy of this licence, visit http://creativecommons.org/licenses/by/4.0/. 
scheme, has been widely adopted to design proper controllers, i.e., the controllers are applied to the systems only at certain moments. That is, the impulsive controllers have a relatively simple structure and are easy to implement and have low cost. Researchers have obtained many valuable results about impulsive control and synchronization in integer-order dynamical networks [21-26, 29, 30,32]. From a practical point of view, a fractional-order network can describe some practical phenomena more accurate than an integer-order model. Therefore, impulsive control is adopted to study the synchronization of fractionalorder dynamical networks as well. In [27], the authors investigated impulsive stabilization and synchronization of fractional-order complex-valued neural networks. In [28], the authors investigated synchronization for a class of fractional-order linear complex networks via impulsive control. In $[27,28]$, some useful synchronization conditions are obtained, from which the impulsive gains and intervals can be calculated for a given network. However, for different networks, it is necessary to calculate the required impulsive intervals and gains repetitively. Therefore, how to design the universal impulsive controllers deserves further studies.

In this paper, we introduce the fractional-order complex-variable dynamical network model and present some preliminaries in Sect. 2. In Sect. 3, we design static and adaptive impulsive controllers, respectively. For static impulsive controllers, we derive the sufficient conditions for achieving synchronization. For adaptive impulsive controllers, we provide the updating laws of the impulsive gains. We perform three numerical examples to verify the results in Sect. 4. In Sect. 5, we give the conclusions.

\section{Model description and preliminaries}

In this section, some definitions and lemma are recalled.

Definition $1([3,6])$ For an integrable function $f(t):\left[t_{0},+\infty\right) \rightarrow \mathbb{R}$, its $\alpha$ th-order fractional integral is defined as

$$
t_{0} I_{t}^{\alpha} f(t)=\frac{1}{\Gamma(\alpha)} \int_{t_{0}}^{t}(t-s)^{\alpha-1} f(s) d s, \quad t \geq t_{0}
$$

where $\Gamma(\cdot)$ stands for the Gamma function and $\alpha>0$.

Definition $2([3,6])$ For function $f \in C^{m}\left(\left[t_{0},+\infty\right), \mathbb{R}\right)$, its $\alpha$ th-order Caputo derivative is defined by

$$
{ }_{t_{0}} D_{t}^{\alpha} f(t)=\frac{1}{\Gamma(m-\alpha)} \int_{t_{0}}^{t}(t-s)^{m-\alpha-1} f^{(m)}(s) d s, \quad t>t_{0},
$$

where $m$ is a positive integer such that $m-1<\alpha<m$.

Lemma 1 ([27]) For $\forall t_{0} \in \mathbb{R}$ and a real-valued continuous function $V(t)$ on $\left[t_{0},+\infty\right)$, if there exists a constant $\theta$ such that

$$
t_{0} D_{t}^{\alpha} V(t) \leq \theta V(t), \quad 0<\alpha<1
$$

then

$$
V(t) \leq V\left(t_{0}\right) e^{\frac{\theta}{\Gamma(\alpha+1)}\left(t-t_{0}\right)^{\alpha}} .
$$


Consider a fractional-order complex-variable dynamical network, described by

$$
{ }_{t_{\sigma}} D_{t}^{\alpha} x_{k}(t)=f\left(x_{k}(t)\right)+b \sum_{l=1}^{N} a_{k l} \Gamma x_{l}(t), \quad t \in\left(t_{\sigma}, t_{\sigma+1}\right], \sigma=0,1,2, \ldots
$$

where $k=1,2, \ldots, N, 0<\alpha<1, x_{k}(t)=\left(x_{k 1}(t), x_{k 2}(t), \ldots, x_{k n}(t)\right)^{T} \in \mathbb{C}^{n}$ is the state variable of node $k, f: \mathbb{C}^{n} \rightarrow \mathbb{C}^{n}$ is a nonlinear complex-valued vector function, $b>0$ is the coupling strength, $\Gamma=\operatorname{diag}\left(\gamma_{1}, \gamma_{2}, \ldots, \gamma_{n}\right)$ is the inner coupling matrix. $A=\left(a_{k l}\right) \in \mathbb{R}^{N \times N}$ is the zerorow-sum outer coupling matrix representing the network topology, defined as: if node $k$ is affected by node $l(k \neq l)$, then $a_{k l} \neq 0$; otherwise, $a_{k l}=0$. The time series $\left\{t_{\sigma}\right\}$ satisfies $0=t_{0}<t_{1}<t_{2}<\cdots<t_{\sigma}<t_{\sigma+1}<\cdots$ and $t_{\sigma} \rightarrow+\infty$ as $\sigma \rightarrow+\infty$.

The network (1) is said to achieve synchronization, if $\lim _{t \rightarrow \infty}\left\|x_{k}(t)-s(t)\right\|=0$, where $s(t)$ is a solution of an isolated node, i.e., $t_{\sigma} D_{t}^{\alpha} s(t)=f(s(t))$ for $t \in\left(t_{\sigma}, t_{\sigma+1}\right]$.

The controlled network with impulsive controllers is written as

$$
\begin{aligned}
& { }_{\sigma} D_{t}^{\alpha} x_{k}(t)=f\left(x_{k}(t)\right)+b \sum_{l=1}^{N} a_{k l} \Gamma x_{l}(t), \quad t \in\left(t_{\sigma}, t_{\sigma+1}\right), \\
& x_{k}\left(t_{\sigma+1}^{+}\right)=x_{k}\left(t_{\sigma+1}^{-}\right)+B\left(t_{\sigma+1}\right)\left(x_{k}\left(t_{\sigma+1}^{-}\right)-s\left(t_{\sigma+1}^{-}\right)\right),
\end{aligned}
$$

where $x_{k}\left(t_{\sigma+1}^{+}\right)=\lim _{t \rightarrow t_{\sigma+1}^{+}} x_{k}(t)$ and $x_{k}\left(t_{\sigma+1}^{-}\right)=\lim _{t \rightarrow t_{\sigma+1}^{-}} x_{k}(t)$. Any solution of (2) satisfies $x_{k}\left(t_{\sigma+1}^{-}\right)=x_{k}\left(t_{\sigma+1}\right) \cdot B\left(t_{\sigma+1}\right) \in(-2,-1) \cup(-1,0)$ is the impulsive gain at $t=t_{\sigma+1}, B\left(t_{0}\right)=0$ and $B(t)=0$ for $t \neq t_{\sigma+1}$.

Assumption 1 Suppose that there exists a positive constant $L$ such that

$$
\begin{aligned}
& (x(t)-s(t))^{T} \overline{(f(x(t))-f(s(t)))}+(f(x(t))-f(s(t)))^{T} \overline{(x(t)-s(t))} \\
& \quad \leq L(x(t)-s(t))^{T} \overline{(x(t)-s(t))}
\end{aligned}
$$

holds for any $x(t), s(t) \in \mathbb{C}^{n}$ and $t>0$.

Throughout this paper, we make Assumption 1. Since the coupling matrix is a zero-rowsum matrix and the impulsive gains $B\left(t_{\sigma+1}\right) \in(-2,-1) \cup(-1,0)$, according to the discussions in Refs. [12] and [46], the existence of the solutions of (3) is guaranteed.

Let $e_{k}(t)=x_{k}(t)-s(t)$, we have the following error system:

$$
\begin{aligned}
& t_{\sigma} D_{t}^{\alpha} e_{k}(t)=f\left(x_{k}(t)\right)-f(s(t))+b \sum_{l=1}^{N} a_{k l} \Gamma e_{l}(t), \quad t \in\left(t_{\sigma}, t_{\sigma+1}\right), \\
& e_{k}\left(t_{\sigma+1}^{+}\right)=e_{k}\left(t_{\sigma+1}^{-}\right)+B\left(t_{\sigma+1}\right) e_{k}\left(t_{\sigma+1}^{-}\right), \quad t=t_{\sigma+1} .
\end{aligned}
$$

\section{Main results}

In what follows, let $e(t)=\left(\left(e_{1}(t)\right)^{T},\left(e_{2}(t)\right)^{T}, \ldots,\left(e_{N}(t)\right)^{T}\right)^{T}, \tau_{\sigma}=t_{\sigma+1}-t_{\sigma}$ be the impulsive intervals, $\lambda$ be the largest eigenvalue of matrix $b\left(A+A^{T}\right) \otimes \Gamma, \theta=\frac{L+\lambda}{\Gamma(\alpha+1)}$, and $\delta(t)=(1+$ $B(t))^{2}$. From the definition of $B(t)$, one has $\delta(t)=1$ for $t \neq t_{\sigma}$. 
Theorem 1 Suppose that Assumption 1 holds. If there exists a constant $\xi>0$ such that

$$
\ln \delta(t)+\xi+\theta \tau_{\sigma}^{\alpha}<0, \quad \sigma=1,2, \ldots
$$

hold, then network (2) achieves synchronization.

Proof Consider the following Lyapunov functional candidate:

$$
V(e(t))=\sum_{k=1}^{N} e_{k}^{T}(t) \overline{e_{k}(t)}, \quad t \in\left(t_{\sigma}, t_{\sigma+1}\right]
$$

When $t \in\left(t_{\sigma}, t_{\sigma+1}\right)$, the derivative of $V(e(t))$ is

$$
\begin{aligned}
t_{\sigma} D_{t}^{\alpha} V(e(t)) \leq & \sum_{k=1}^{N} e_{k}^{T}(t)\left({ }_{t_{\sigma}} D_{t}^{\alpha} \overline{\left.e_{k}(t)\right)}+\sum_{k=1}^{N}\left({ }_{t_{\sigma}} D_{t}^{\alpha} e_{k}^{T}(t)\right) \overline{e_{k}(t)}\right. \\
= & \sum_{k=1}^{N}\left(e_{k}^{T}(t) \overline{\left(f\left(x_{k}(t)-f(s(t))\right)\right.}+\left(f\left(x_{k}(t)-f(s(t))\right)^{T} \overline{e_{k}(t)}\right)\right. \\
& +b \sum_{k=1}^{N} \sum_{l=1}^{N}\left(e_{l}^{T}(t) a_{k l} \Gamma \overline{e_{k}(t)}+e_{k}^{T}(t) a_{k l} \Gamma \overline{e_{l}(t)}\right) \\
\leq & L e^{T}(t) \overline{e(t)}+b e^{T}(t)\left(A+A^{T}\right) \otimes \Gamma \overline{e(t)} \\
\leq & (L+\lambda) V(e(t))
\end{aligned}
$$

which gives

$$
\begin{aligned}
V(e(t)) & \leq V\left(e\left(t_{\sigma}^{+}\right)\right) e^{\frac{L+\lambda}{\Gamma(\alpha+1)}\left(t-t_{\sigma}\right)^{\alpha}} \\
& =V\left(e\left(t_{\sigma}^{+}\right)\right) e^{\theta\left(t-t_{\sigma}\right)^{\alpha}} .
\end{aligned}
$$

When $t=t_{\sigma+1}$, one has

$$
\begin{aligned}
V\left(e\left(t_{\sigma+1}^{+}\right)\right) & =\sum_{k=1}^{N} e_{k}^{T}\left(t_{\sigma+1}^{+}\right) \overline{e_{k}\left(t_{\sigma+1}^{+}\right)} \\
& =\left(1+B\left(t_{\sigma+1}\right)\right)^{2} \sum_{k=1}^{N} e_{k}^{T}\left(t_{\sigma+1}^{-}\right) \overline{e_{k}\left(t_{\sigma+1}^{-}\right)} \\
& =\delta\left(t_{\sigma+1}\right) V\left(e\left(t_{\sigma+1}^{-}\right)\right) .
\end{aligned}
$$

When $\sigma=0$, from (5) and (6),

$$
\begin{aligned}
& V\left(e\left(t_{1}^{-}\right)\right) \leq V\left(e\left(t_{0}\right)\right) \exp \left(\theta \tau_{1}^{\alpha}\right), \\
& V\left(e\left(t_{1}^{+}\right)\right) \leq \delta\left(t_{1}\right) V\left(e\left(t_{1}^{-}\right)\right) \leq \delta\left(t_{1}\right) V\left(e\left(t_{0}\right)\right) \exp \left(\theta \tau_{1}^{\alpha}\right) .
\end{aligned}
$$

When $\sigma=1$,

$$
V\left(e\left(t_{2}^{-}\right)\right) \leq V\left(e\left(t_{1}\right)\right) \exp \left(\theta \tau_{2}^{\alpha}\right) \leq \delta\left(t_{1}\right) V\left(e\left(t_{0}\right)\right) \exp \left(\theta\left(\tau_{1}^{\alpha}+\tau_{2}^{\alpha}\right)\right)
$$




$$
\begin{aligned}
V\left(e\left(t_{2}^{+}\right)\right) & \leq \delta\left(t_{2}\right) V\left(e\left(t_{2}^{-}\right)\right) \\
& \leq \delta\left(t_{2}\right) \delta\left(t_{1}\right) V\left(e\left(t_{0}\right)\right) \exp \left(\theta\left(\tau_{1}^{\alpha}+\tau_{2}^{\alpha}\right)\right) \\
& =V\left(e\left(t_{0}\right)\right) \prod_{\epsilon=1}^{2} \delta\left(t_{\epsilon}\right) \exp \left(\theta \tau_{\epsilon}^{\alpha}\right) .
\end{aligned}
$$

By induction,

$$
V\left(e\left(t_{\sigma}^{+}\right)\right) \leq V\left(e\left(t_{0}\right)\right) \prod_{\epsilon=1}^{\sigma} \delta\left(t_{\epsilon}\right) \exp \left(\theta \tau_{\epsilon}^{\alpha}\right), \quad \sigma=1,2, \ldots
$$

From inequalities (4),

$$
\delta\left(t_{\epsilon}\right) \exp \left(\theta \tau_{\epsilon}^{\alpha}\right) \leq \exp (-\xi), \quad \epsilon=1,2, \ldots
$$

and

$$
V\left(e\left(t_{\sigma}^{+}\right)\right) \leq V\left(e\left(t_{0}\right)\right) \exp (-\sigma \xi)
$$

That is, $V\left(e\left(t_{\sigma}^{+}\right)\right) \rightarrow 0$ when $\sigma \rightarrow+\infty$. Therefore, when $t \in\left(t_{\sigma}, t_{\sigma+1}\right]$,

$$
V(e(t)) \leq V\left(e\left(t_{\sigma}^{+}\right)\right) \exp \left(\theta\left(t-t_{\sigma}\right)^{\alpha}\right)
$$

i.e., $V(e(t)) \rightarrow 0$ and $\left\|e_{k}(t)\right\| \rightarrow 0$ as $t \rightarrow+\infty$. This completes the proof.

Remark 1 By simple calculations, we can estimate the positive constant $\theta$ in Theorem 1 , and then calculate the impulsive gains from conditions (4). However, for different networks, we must repeatedly calculate the impulsive gains. Therefore, we design adaptive impulsive controllers to avoid this situation.

Theorem 2 Suppose that Assumption 1 holds. If there exists a constant $\xi>0$ such that the following conditions:

$$
\ln \delta\left(t_{\sigma}\right)+\xi+\widehat{\theta}\left(t_{\sigma}\right) \tau^{\alpha}<0, \quad \sigma=0,1,2, \ldots
$$

hold, where $\widehat{\theta}(t)$ is the estimated value of $\theta, t_{\sigma} D_{t}^{\alpha} \widehat{\theta}(t)=\Gamma(\alpha+1) \omega \sum_{k=1}^{N} e_{k}^{T}(t) \overline{e_{k}(t)}, t \in$ $\left(t_{\sigma}, t_{\sigma+1}\right]$ and $\omega>0$ is a positive constant, then the controlled network (2) achieves synchronization.

Proof Consider the following Lyapunov function:

$$
V(e(t))=\sum_{k=1}^{N} e_{k}^{T}(t) \overline{e_{k}(t)}+\frac{\delta(t)}{2 \omega}(\widehat{\theta}(t)-\theta)^{2}, \quad t \in\left(t_{\sigma}, t_{\sigma+1}\right]
$$

When $t \in\left(t_{\sigma}, t_{\sigma+1}\right)$, the function $V(e(t))$ can be written as

$$
V(e(t))=\sum_{k=1}^{N} e_{k}^{T}(t) \overline{e_{k}(t)}+\frac{1}{2 \omega}(\widehat{\theta}(t)-\theta)^{2}
$$


and the derivative of $V(e(t))$ can be calculated as

$$
\begin{aligned}
t_{\sigma} D_{t}^{\alpha} V(e(t)) \leq & \sum_{k=1}^{N}\left(t_{\sigma} D_{t}^{\alpha} e_{k}^{T}(t)\right) \overline{e_{k}(t)}+\sum_{k=1}^{N} e_{k}^{T}(t)\left(t_{\sigma} D_{t}^{\alpha} \overline{e_{k}(t)}\right) \\
& +\frac{1}{\omega}(\widehat{\theta}(t)-\theta)_{t_{\sigma}} D_{t}^{\alpha} \widehat{\theta}(t) \\
= & \sum_{k=1}^{N}\left(e_{k}^{T}(t) \overline{\left(f\left(x_{k}(t)\right)-f(s(t))\right)}+\left(f\left(x_{k}(t)\right)-f(s(t))\right)^{T} \overline{\left.e_{k}(t)\right)}\right. \\
& +b \sum_{k=1}^{N} \sum_{l=1}^{N}\left(e_{l}^{T}(t) a_{k l} \Gamma \overline{e_{k}(t)}+e_{k}^{T}(t) a_{k l} \Gamma \overline{\left.e_{l}(t)\right)}\right. \\
& +\Gamma(\alpha+1)(\widehat{\theta}(t)-\theta) \sum_{k=1}^{N} e_{k}^{T}(t) \overline{e_{k}(t)} \\
\leq & \Gamma(\alpha+1) \widehat{\theta}(t) e^{T}(t) \overline{e(t)} \\
\leq & \Gamma(\alpha+1) \widehat{\theta}\left(t_{\sigma+1}\right) V(e(t))
\end{aligned}
$$

which gives

$$
V(e(t)) \leq V\left(e\left(t_{\sigma}^{+}\right)\right) \exp \left(\widehat{\theta}\left(t_{\sigma+1}\right)\left(t-t_{\sigma}\right)^{\alpha}\right) .
$$

When $t=t_{\sigma+1}$, one has

$$
\begin{aligned}
V\left(e\left(t_{\sigma+1}^{+}\right)\right) & =\sum_{k=1}^{N} e_{k}^{T}\left(t_{\sigma+1}^{+}\right) \overline{e_{k}\left(t_{\sigma+1}^{+}\right)}+\frac{\delta\left(t_{\sigma+1}\right)}{2 \omega}(\widehat{\theta}(t)-\theta)^{2} \\
& =\left(1+B\left(t_{\sigma+1}\right)\right)^{2} \sum_{k=1}^{N} e_{k}^{T}\left(t_{\sigma+1}^{-}\right) \overline{e_{k}\left(t_{\sigma+1}^{-}\right)}+\frac{\delta\left(t_{\sigma+1}\right)}{2 \omega}(\widehat{\theta}(t)-\theta)^{2} \\
& =\delta\left(t_{\sigma+1}\right)\left(\sum_{k=1}^{N} e_{k}^{T}\left(t_{\sigma+1}^{-}\right) \overline{e_{k}\left(t_{\sigma+1}^{-}\right)}+\frac{1}{2 \omega}(\widehat{\theta}(t)-\theta)^{2}\right) \\
& =\delta\left(t_{\sigma+1}\right) V\left(e\left(t_{\sigma+1}^{-}\right)\right) .
\end{aligned}
$$

Therefore, similar to the proof of Theorem 1, the proof is completed.

Remark 2 When $\tau_{\sigma}$ and $\xi$ are fixed, we choose

$$
-\exp \left(-\frac{\xi+\widehat{\theta}\left(t_{\sigma}\right) \tau_{\sigma}}{2}\right)-1+\varepsilon \leq B\left(t_{\sigma}\right) \leq \exp \left(-\frac{\xi+\widehat{\theta}\left(t_{\sigma}\right) \tau_{\sigma}}{2}\right)-1-\varepsilon
$$

such that the conditions (7) is satisfied, where $\varepsilon>0$ is an arbitrary constant.

\section{Numerical illustrations}

Example 1 Choose the node dynamics as the fractional-order complex-variable Lorenz system [47]

$$
t_{\sigma} D_{t}^{\alpha} x_{k 1}(t)=a\left(x_{k 2}(t)-x_{k 1}(t)\right)
$$



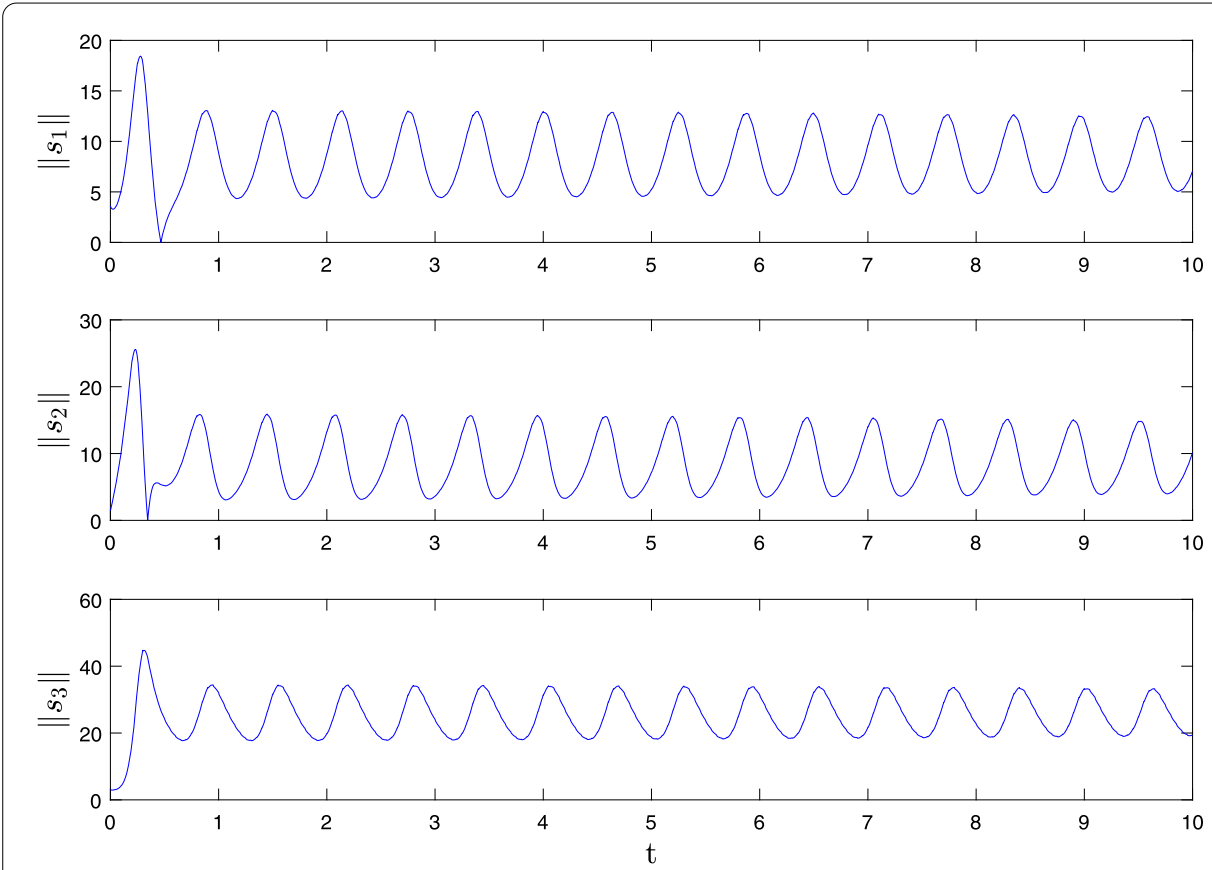

Figure 1 The orbits of $\left\|s_{k}(t)\right\|$ for fractional-order complex-variable Lorenz system with $s(0)=(3+2 j ; 1+1 j ; 3)^{T}$

$$
\begin{aligned}
& t_{\sigma} D_{t}^{\alpha} x_{k 2}(t)=b x_{k 1}(t)-x_{k 2}(t)-x_{k 1}(t) x_{k 3}(t), \\
& t_{\sigma} D_{t}^{\alpha} x_{k 3}(t)=\frac{1}{2}\left(\overline{x_{k 1}(t)} x_{k 2}(t)+x_{k 1}(t) \overline{x_{k 2}(t)}\right)-c x_{k 3}(t),
\end{aligned}
$$

where $x_{k 1}$ and $x_{k 2}$ are complex variables, $x_{k 3}$ is real variable, which is chaotic when the system parameters are chosen as $a=10, b=28, c=8 / 3, \alpha=0.995$ and $\tau_{\sigma}=0.05 \sigma$. Figure 1 shows the orbits of $\left\|s_{k}(t)\right\|$ with $s(0)=(3+2 j, 1+j, 3)^{T}$ and $j=\sqrt{-1}$. From Fig. 1 , there exist three constants $M_{1}=19, M_{2}=26, M_{3}=47$ such that $\left\|s_{1}\right\| \leq M_{1},\left\|s_{2}\right\| \leq M_{2},\left\|s_{3}\right\| \leq M_{3}$. Therefore, one has

$$
\begin{aligned}
\left(x_{k}(\right. & t)-s(t))^{T} \overline{\left(f\left(x_{k}\right)-f(s)\right)}+\left(f\left(x_{k}\right)-f(s)\right)^{T} \overline{\left(x_{k}(t)-s(t)\right)} \\
= & -2 a e_{k 1} \overline{e_{k 1}}-2 e_{k 2} \overline{e_{k 2}}-2 c e_{k 3}^{2}+\left(a+b-s_{3}\right)\left(e_{k 1} \overline{e_{k 2}}+\overline{e_{k 1}} e_{k 2}\right) \\
& +s_{2}\left(\overline{e_{k 1}} e_{k 3}+e_{k 1} e_{k 3}\right) \\
\leq & -2 a e_{k 1} \overline{e_{k 1}}-2 e_{k 2} \overline{e_{k 2}}-2 c e_{k 3}^{2}+\left(a+b+M_{3}\right)\left(e_{k 1} \overline{e_{k 2}}+\overline{e_{k 1}} e_{k 2}\right) \\
& +M_{2}\left(\overline{e_{k 1}} e_{k 3}+e_{k 1} e_{k 3}\right) \\
\leq & \left(-2 a+\left(a+b+M_{3}\right) \mu+M_{2} v\right) e_{k 1} \overline{e_{k 1}}+\left(-2+\left(a+b+M_{3}\right) \mu^{-1}\right) e_{k 2} \overline{e_{k 2}} \\
& +\left(-2 c+M_{2} v^{-1}\right) e_{k 3}^{2},
\end{aligned}
$$

where $\mu>0, v>0$. Choosing $\mu=1.05, v=0.31$ gives $L=79$ in Assumption 1 . 
Consider the synchronization of network (2) with 10 nodes. Choose $b=0.5, \Gamma=$ $\operatorname{diag}(1,1,1)$ and

$$
A=\left[\begin{array}{cccccccccc}
-3 & 1 & 0 & 2 & 0 & -2 & 1 & 0 & 0 & 1 \\
0 & -6 & 0 & 1 & 0 & 1 & 2 & 1 & 0 & 1 \\
-2 & 1 & -5 & 0 & 1 & 2 & 0 & 2 & 0 & 1 \\
1 & 1 & 0 & -4 & 1 & 0 & -2 & 0 & 3 & 0 \\
0 & 1 & -1 & 0 & -6 & 0 & 1 & 2 & 1 & 2 \\
0 & 1 & 2 & 1 & 0 & -5 & 0 & -1 & 0 & 2 \\
2 & 0 & 0 & 2 & 0 & 2 & -7 & 0 & 0 & 1 \\
0 & -2 & 3 & 0 & 2 & 0 & 1 & -4 & 0 & 0 \\
1 & 0 & 3 & 0 & -1 & 1 & 0 & 0 & -4 & 0 \\
0 & 1 & 0 & 1 & -2 & 0 & 0 & 2 & 1 & -3
\end{array}\right],
$$

which gives $\lambda=0.4693$. In numerical simulations, choose $\xi=0.001$, the impulsive gains $B\left(t_{\sigma}\right)=-0.9, \sigma=1,2, \ldots$, one has $\delta\left(t_{\sigma}\right)=0.01$. By simple calculations, we have $\ln \delta(t)+\xi+$ $\theta \tau_{\sigma}^{\alpha}=-0.5707<0$, i.e., the conditions (4) hold and the synchronization can be achieved. The initial values of $s(t)$ and $x_{k}(t)$ are chosen randomly. Figure 2 shows the orbits of the real and imaginary parts of $x_{k l}(t)$ and $s_{l}(t), k=1,2, \ldots, 10, l=1,2,3$. The superscripts $r$ and $i$ denote the real parts and the imaginary parts, respectively.

Example 2 Consider the above network in Example 1 via the adaptive impulsive controllers. Choose $\omega=0.01, \xi=0.001$ and $\widehat{\theta}(0)=1$. According to Remark 2, choose

$$
B\left(t_{\sigma}\right)=\exp \left(-\frac{\xi+\widehat{\theta}\left(t_{\sigma}\right) \tau_{\sigma}}{2}\right)-1-\varepsilon
$$
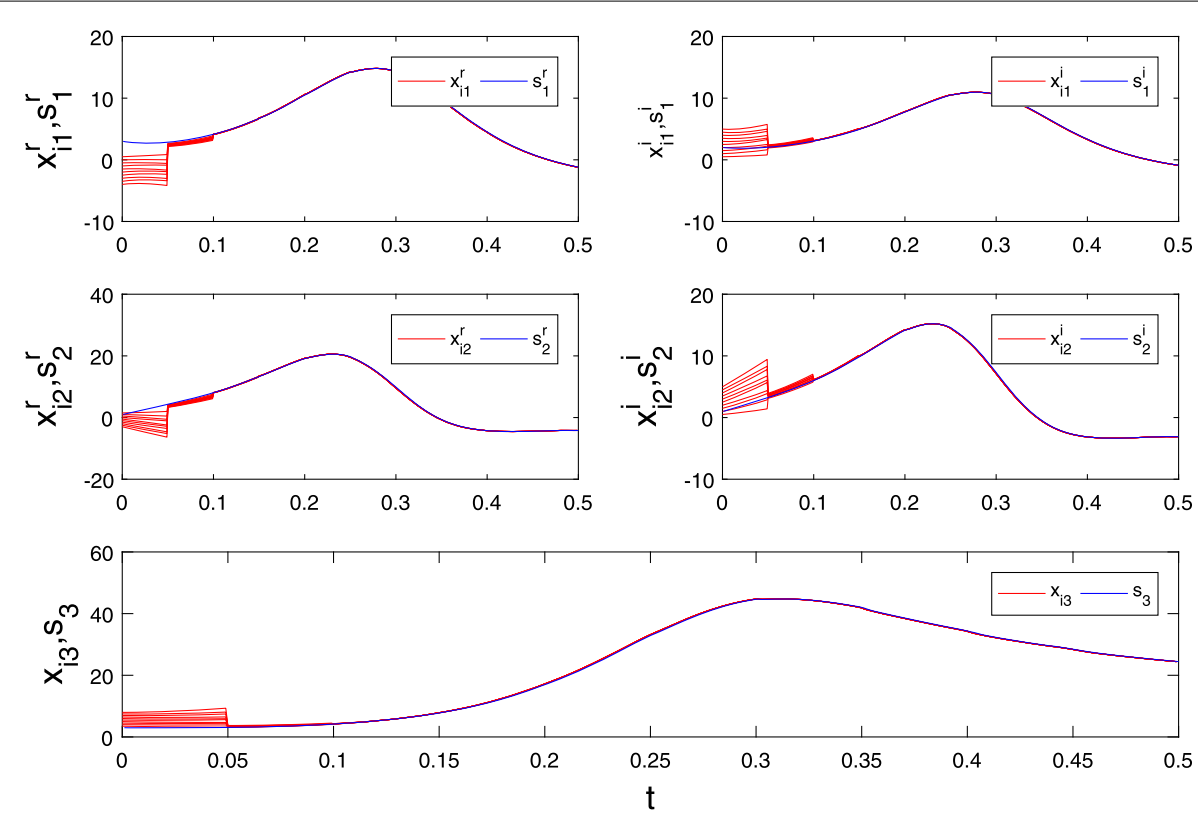

Figure 2 The orbits of the real and imaginary parts of $x_{k l}(t)$ and $s_{/}(t)$ 
with $\varepsilon=0.001$. Figure 3 shows the orbits of the real and imaginary parts of $x_{k l}(t)$ and $s_{l}(t)$, $k=1,2, \ldots, 10, l=1,2,3$. Figure 4 shows the impulsive gains $B\left(t_{\sigma}\right)$.
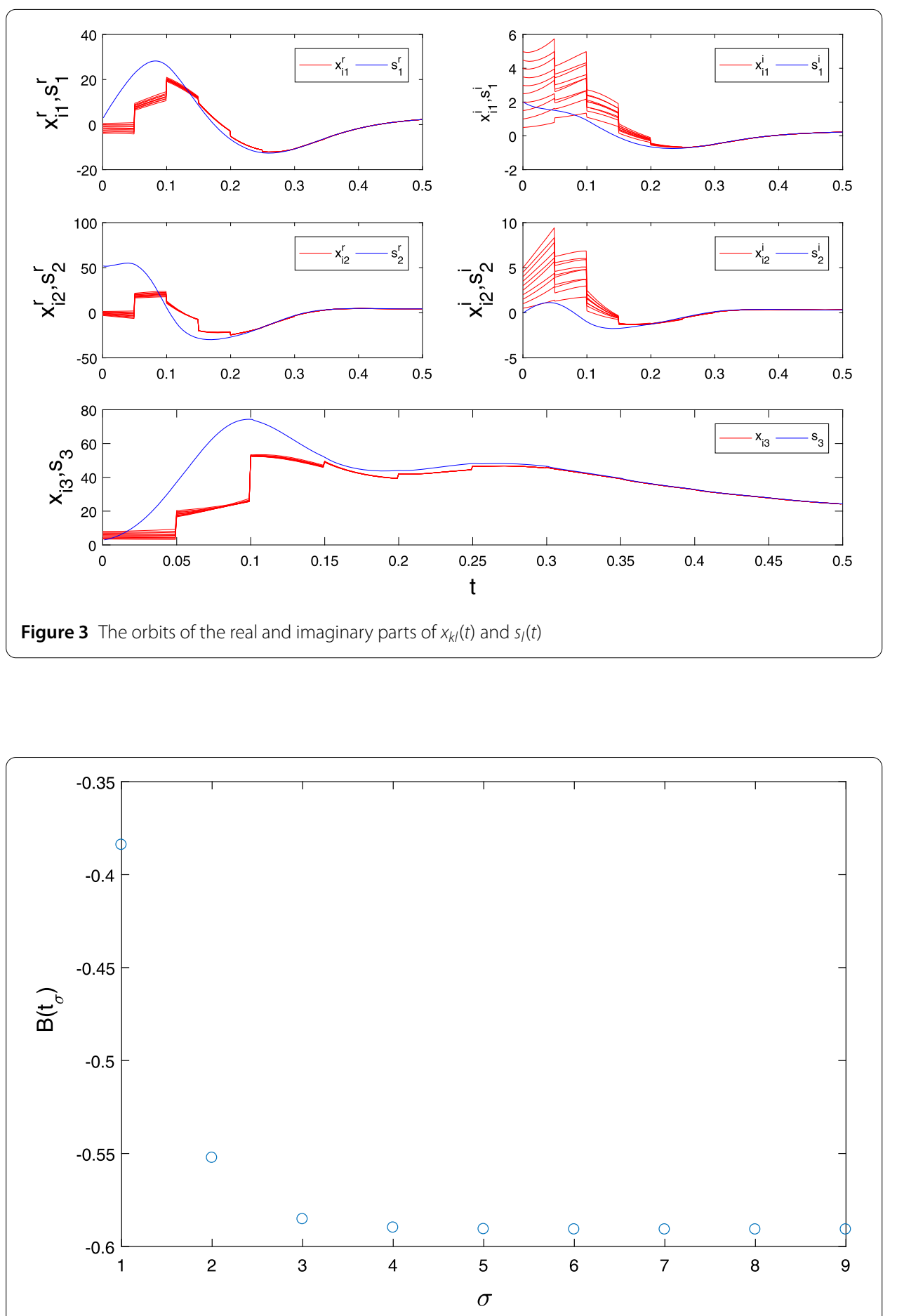

Figure 4 The impulsive gains $B\left(t_{\sigma}\right)$ versus $\sigma$ 

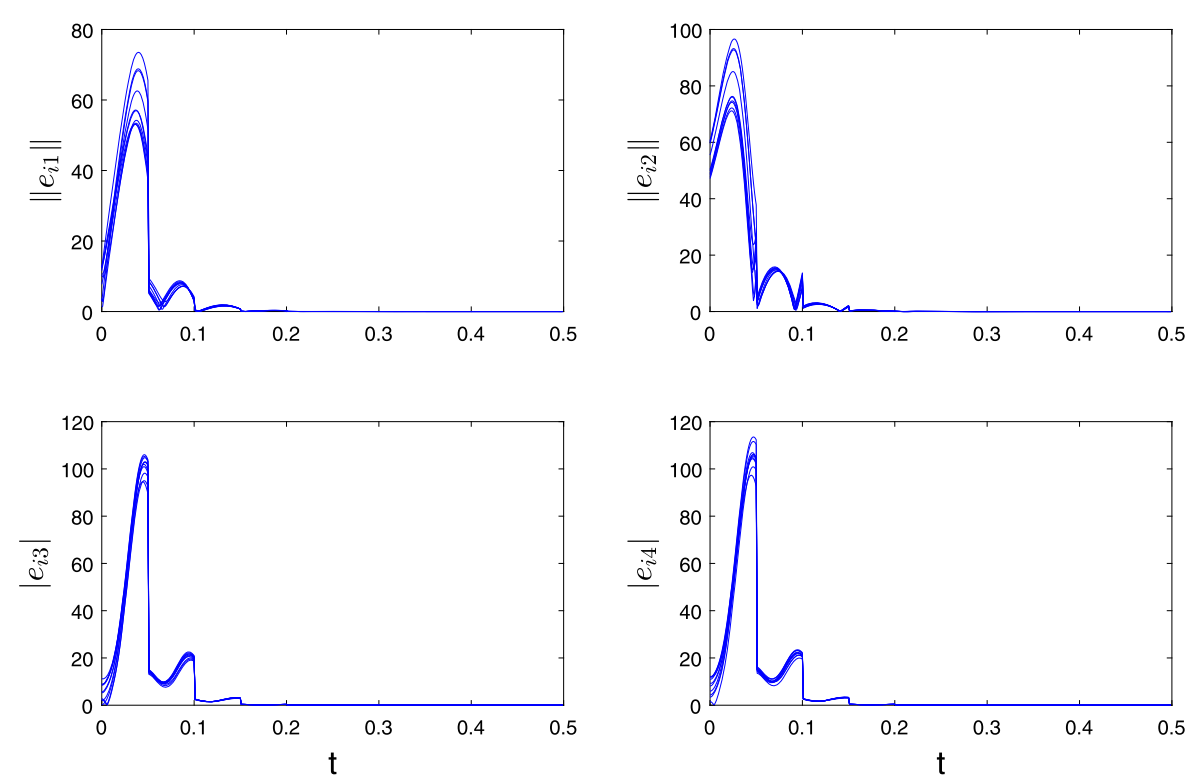

Figure 5 The orbits of $\left\|e_{k l}(t)\right\|$

Example 3 Choose the node dynamics as fractional-order hyperchaotic complex Lü system [48]

$$
\begin{aligned}
& D_{t_{0}}^{\alpha} x_{k 1}=42\left(x_{k 2}-x_{k 1}\right)+x_{k 4}, \\
& D_{t_{0}}^{\alpha} x_{k 2}=25 x_{k 2}+x_{k 4}-x_{k 1} x_{k 3}, \\
& D_{t_{0}}^{\alpha} x_{k 3}=\frac{1}{2}\left(\overline{x_{k 1}} x_{k 2}+x_{k 1} \overline{x_{k 2}}\right)-6 x_{k 3}, \\
& D_{t_{0}}^{\alpha} x_{k 4}=\frac{1}{2}\left(\overline{x_{k 1}} x_{k 2}+x_{k 1} \overline{x_{k 2}}\right)-5 x_{k 4},
\end{aligned}
$$

where $k=1,2, \ldots, N, x_{k 1}$ and $x_{k 2}$ are complex variables, $x_{k 3}$ and $x_{k 4}$ are real variables, $\alpha=$ 0.995. Choose the same $A$ as Example 1, the initial values $s(0)=(3+2 j, 1+j, 3,4)^{T}$ and $x_{k}(t)$ are chosen randomly.

Choose $\tau_{\sigma}=0.2, \omega=0.001, \xi=0.001$ and $\widehat{\theta}(0)=0.1$. According to Remark 2, choose

$$
B\left(t_{\sigma}\right)=\exp \left(-\frac{\xi+\widehat{\theta}\left(t_{\sigma}\right) \tau_{\sigma}}{2}\right)-1-\varepsilon
$$

with $\varepsilon=0.001$. Figure 5 shows the orbits of the real and imaginary parts of $x_{k l}(t)$ and $s_{l}(t)$, $k=1,2, \ldots, 10, l=1,2,3$. Figure 6 shows the impulsive gains $B\left(t_{\sigma}\right)$.

From Examples 2 and 3, the impulsive gains need not be calculated in advance for different networks. And they can adjust themselves to the required values according to the updating laws. That is, the adaptive impulsive controllers are universal to some extent.

\section{Conclusions}

Both static and adaptive impulsive controllers were designed. Two corresponding synchronization conditions were derived as well. Particularly, for the adaptive impulsive con- 


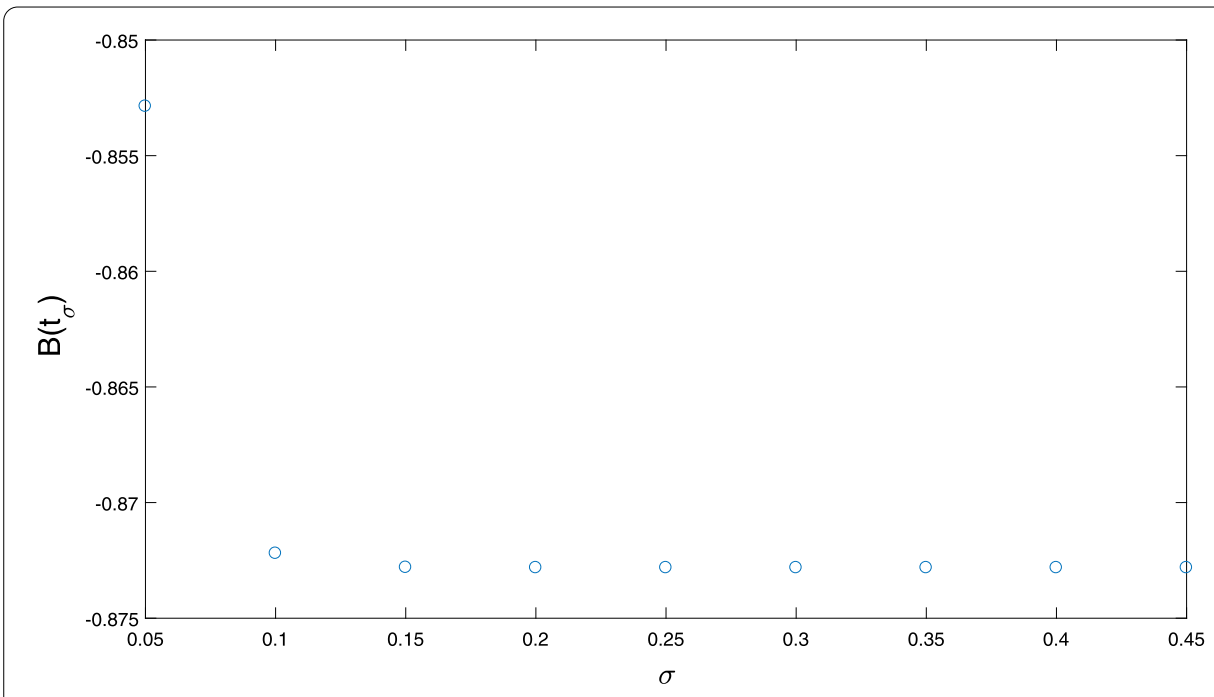

Figure 6 The impulsive gains $B\left(t_{\sigma}\right)$ versus $\sigma$

trollers, the updating law for the impulsive gains was provided. Examples 2 and 3 demonstrated the points well and implied that the adaptive impulsive controllers are universal for different networks.

\section{Acknowledgements}

The authors are thankful to the editor and anonymous reviewers for their insightful comments and suggestions, which strengthened our manuscript.

\section{Funding}

This work is jointly supported by the National Natural Science Foundation of China (61463022 and 61963019), the NSF for Distinguished Young Scholar of Jiangxi Province of China (20171BCB23031) and the Graduate Innovation Project of Jiangxi Province of China (YC2020-S187).

\section{Availability of data and materials}

Not applicable.

\section{Competing interests}

The authors declare that they have no competing interests.

\section{Authors' contributions}

All the authors contributed equally to this work. They all read and approved the final version of the manuscript.

\section{Publisher's Note}

Springer Nature remains neutral with regard to jurisdictional claims in published maps and institutional affiliations.

Received: 20 February 2021 Accepted: 8 July 2021 Published online: 10 August 2021

\section{References}

1. Ahmed, E., Elgazzar, A.: On fractional order differential equations model for nonlocal epidemics. Physica A 379, 607-614 (2007)

2. Butzer, P., Westphal, U.: An Introduction to Fractional Calculus. World Scientific, Singapore (2000)

3. Podlubny, l.: Fractional Differential Equations. Academic Press, New York (1999)

4. Hiffer, R.: Applications of Fractional Calculus in Physics. World Scientific, Singapore (2000)

5. Laskin, N.: Fractional quantum mechanics and Lévy path integrals. Phys. Lett. A 268, 298-305 (2000)

6. Kilbas, A., Srivastava, H., Trujillo, J.: Theory and Applications of Fractional Differential Equations. Elsevier, Amsterdam (2006)

7. Yu, F., Liu, L., He, B., Huang, Y., Shi, C., Cai, S., Song, Y., Du, S., Wan, Q.: Analysis and FPGA realization of a novel 5D hyperchaotic four-wing memristive system, active control synchronization, and secure communication application. Complexity 2019, 4047957 (2019)

8. Yu, F., Liu, L., Shen, H., Zhang, Z., Huang, Y., Shi, C., Cai, S., Wu, X., Du, S., Wan, Q.: Dynamic analysis, circuit design, and synchronization of a novel $6 \mathrm{D}$ memristive four-wing hyperchaotic system with multiple coexisting attractors. Complexity 2020, 5904607 (2020) 
9. Yu, F., Qian, S., Chen, X., Huang, Y., Liu, L., Shi, C., Cai, S., Song, Y., Wang, C.: A new 4D four-wing memristive hyperchaotic system: dynamical analysis, electronic circuit design, shape synchronization and secure communication. Int. J. Bifurc. Chaos 30, 2050147 (2020)

10. Tan, W., Jiang, F., Huang, C., Zhou, L.: Synchronization for a class of fractional-order hyperchaotic system and its application. J. Appl. Math. 2012, 974639 (2012)

11. Chen, W.: Nonlinear dynamics and chaos in a fractional order financial system. Chaos Solitons Fractals 36, 1305-1314 (2008)

12. Chen, F., Chen, A., Wang, X.: On the solutions for impulsive fractional functional differential equations. Differ. Equ. Dyn. Syst. 17, 379-391 (2009)

13. Xu, C., Liao, M., Li, P., Guo, Y., Xiao, Q., Yuan, S.: Influence of multiple time delays on bifurcation of fractional-order neural networks. Appl. Math. Comput. 361, 565-582 (2019)

14. Pratap, A., Raja, R., Cao, J., Huang, C., Niezabitowski, M., Bagdasar, O.: Stability of discrete-time fractional-order time-delayed neural networks in complex field. Math. Methods Appl. Sci. 44, 419-440 (2020)

15. Ren, G., Xu, Y., Wang, C.: Synchronization behavior of coupled neuron circuits composed of memristors. Nonlinear Dyn. 88, 893-901 (2017)

16. Wu, Z., Wang, H.: Impulsive pinning synchronization of discrete-time network. Adv. Differ. Equ. 2016, 36 (2016)

17. Rao, P., Wu, Z., Liu, M.: Adaptive projective synchronization of dynamical networks with distributed time delays. Nonlinear Dyn. 67, 1729-1736 (2012)

18. Liu, M., Jiang, H., Hu, C.: Finite-time synchronization of delayed dynamical networks via aperiodically intermittent control. J. Franklin Inst. 354, 5374-5397 (2017)

19. Gong, X., Wu, Z:: Adaptive pinning impulsive synchronization of dynamical networks with time-varying delay. Adv. Differ. Equ. 2015, 240 (2015)

20. Zhou, P., Cai, S.: Adaptive exponential lag synchronization for neural networks with mixed delays via intermittent control. Adv. Differ. Equ. 2018, 40 (2018)

21. Lu, J., Wang, Z., Cao, J., Ho, D., Kurths, J.: Pinning impulsive stabilization of nonlinear dynamical networks with time-varying delay. Int. J. Bifurc. Chaos 22, 1250176 (2012)

22. $\mathrm{Wu}, \mathrm{Z}$., Liu, D., Ye, Q.: Pinning impulsive synchronization of complex-variable dynamical network. Commun. Nonlinear Sci. Numer. Simul. 20, 273-280 (2015)

23. Leng, H., Wu, Z:: Impulsive synchronization of complex-variable network with distributed time delays. Physica A 536, 122602 (2019)

24. Mahdavi, N., Menhaj, M., Kurths, J., Lu, J., Afshar, A.: Pinning impulsive synchronization of complex dynamical networks. Int. J. Bifurc. Chaos 22, 1250239 (2012)

25. Lu, J., Kurths, J., Cao, J., Mahdavi, N., Huang, C.: Synchronization control for nonlinear stochastic dynamical networks: pinning impulsive strategy. IEEE Trans. Neural Netw. Learn. Syst. 23, 285-292 (2012)

26. Lu, J., Ho, D., Cao, J., Kurths, J.: Single impulsive controller for globally exponential synchronization of dynamical networks. Nonlinear Anal., Real World Appl. 14, 581-593 (2013)

27. Wan, P., Jian, J.: Impulsive stabilization and synchronization of fractional-order complex-valued neural networks, Neural Process. Lett. 50, 2201-2218 (2019)

28. Liu, N., Fang, J., Deng, W.: Synchronization for a class of fractional-order linear complex networks via impulsive control. Int. J. Control. Autom. Syst. 16, 2839-2844 (2018)

29. Li, C.J., Yu, X.H., Liu, Z.W., Huang, T.W.: Asynchronous impulsive containment control in switched multi-agent systems. Inf. Sci. 370-371, 667-679 (2016)

30. Wu, Z., Leng, H.: Impulsive synchronization of drive-response chaotic delayed neural networks. Adv. Differ. Equ. 2016, 206 (2016)

31. Li, H., Hu, C., Jiang, Y., Wang, Z., Teng, Z.: Pinning adaptive and impulsive synchronization of fractional-order complex dynamical networks. Chaos Solitons Fractals 92, 142-149 (2016)

32. Zhao, H., Li, L., Peng, H., Xiao, J., Yang, Y., Zheng, M.: Impulsive control for synchronization and parameters identification of uncertain multi-links complex network. Nonlinear Dyn. 83, 1437-1451 (2016)

33. Song, C., Fei, S., Cao, J., Huang, C.: Robust synchronization of fractional-order uncertain chaotic systems based on output feedback sliding mode control. Mathematics 7, 599 (2019)

34. Zhou, Y., Wan, X., Huang, C., Yang, X.: Finite-time stochastic synchronization of dynamic networks with nonlinear coupling strength via quantized intermittent control. Appl. Math. Comput. 376, 125157 (2020)

35. Pratap, A., Raja, R., Cao, J., Alzabut, J., Huang, C.: Finite-time synchronization criterion of graph theory perspective fractional-order coupled discontinuous neural networks. Adv. Differ. Equ. 2020, 97 (2020)

36. Chen, D., Zhang, W., Cao, J., Huang, C.: Fixed time synchronization of delayed quaternion-valued memristor-based neural networks. Adv. Differ. Equ. 2020, 92 (2020)

37. Pratap, A., Raja, R., Alzabut, J., Cao, J., Rajchakit, G., Huang, C.: Mittag-Leffler stability and adaptive impulsive synchronization of fractional order neural networks in quaternion field. Math. Methods Appl. Sci. 43, 6223-6253 (2020)

38. Liu, X., Chen, T: Synchronization of nonlinear coupled networks via aperiodically intermittent pinning control. IEEE Trans. Neural Netw. Learn. Syst. 26, 113-126 (2015)

39. Liu, X., Chen, T.: Synchronization of linearly coupled networks with delays via aperiodically intermittent pinning control. IEEE Trans. Neural Netw. Learn. Syst. 26, 2396-2407 (2015)

40. Cai, S., Lei, X., Liu, Z.: Outer synchronization between two hybrid-coupled delayed dynamical networks via aperiodically adaptive intermittent pinning control. Complexity 21, 593-605 (2016)

41. Zhang, J., Wang, Y., Ma, Z., Qiu, J., Alsaadi, F.: Intermittent control for cluster-delay synchronization in directed networks. Complexity 2018, 1069839 (2018)

42. Zhou, P., Cai, S.: Pinning synchronization of complex directed dynamical networks under decentralized adaptive strategy for aperiodically intermittent control. Nonlinear Dyn. 90, 287-299 (2017)

43. Wu, X., Feng, J., Nie, Z:: Pinning complex-valued complex network via aperiodically intermittent control. Neurocomputing 305, 70-77 (2018)

44. Novicenko, V: Delayed feedback control of synchronization in weakly coupled oscillator networks. Phys. Rev. E 92 $022919(2015)$ 
45. Tang, S., Pang, W., Cheke, R., Wu, J.: Global dynamics of a state-dependent feedback control system. Adv. Differ. Equ. 2015, 322 (2015)

46. Zhang, T., Xiong, L.: Periodic motion for impulsive fractional functional differential equations with piecewise Caputo derivative. Appl. Math. Lett. 101, 106072 (2020)

47. Luo, C., Wang, X.: Chaos in the fractional-order complex Lorenz system and its synchronization. Nonlinear Dyn. 71, 241-257 (2013)

48. Yang, L., Jiang, J.: Complex dynamical behavior and modified projective synchronization in fractional-order hyper-chaotic complex Lü system. Chaos Solitons Fractals 78, 267-276 (2015)

Submit your manuscript to a SpringerOpen ${ }^{\circ}$ journal and benefit from:

- Convenient online submission

$\checkmark$ Rigorous peer review

- Open access: articles freely available online

- High visibility within the field

- Retaining the copyright to your article

Submit your next manuscript at $\gg$ springeropen.com 